\title{
High Concentrations of Serum Soluble E-Cadherin in Patients With Q Fever
}

\author{
Soraya Mezouar ${ }^{1}$, Ikram Omar Osman ${ }^{1}$, Cléa Melenotte ${ }^{1}$, Camélia Slimani ${ }^{1}$, \\ Céline Chartier ${ }^{2}$, Didier Raoult ${ }^{1}$, Jean-Louis Mege ${ }^{1,2}$ and Christian A. Devaux ${ }^{1,3 *}$ \\ ${ }^{1}$ Aix-Marseille Univ, IRD, APHM, MEPHI, IHU-Méditerranée Infection, Marseille, France, ${ }^{2}$ APHM, IHU-Méditerranée Infection, \\ UF Immunologie, Marseille, France, ${ }^{3} \mathrm{CNRS}$, Marseille, France
}

OPEN ACCESS

Edited by:

Daniel E. Voth,

University of Arkansas for Medical

Sciences, United States

Reviewed by:

Guoquan Zhang,

University of Missouri, United States

Gillbert Kersh

Centers for Disease Control and

Prevention (CDC), United States

*Correspondence:

Christian A. Devaux

christian.devaux@

mediterranee-infection.com

Specialty section:

This article was submitted to

Bacteria and Host

a section of the journal

Frontiers in Cellular and Infection

Microbiology

Received: 27 March 2019 Accepted: 07 June 2019

Published: 21 June 2019

Citation:

Mezouar S, Omar Osman I, Melenotte C, Slimani C, Chartier C,

Raoult D, Mege J-L and Devaux CA (2019) High Concentrations of Serum

Soluble E-Cadherin in Patients

With Q Fever.

Front. Cell. Infect. Microbiol. 9:219.

doi: 10.3389/fcimb.2019.00219
Cadherins switching is a hallmark of neoplasic processes. The E-cadherin (E-cad) subtype is one of the surface molecules regulating cell-to-cell adhesion. After its cleavage by sheddases, a soluble fragment (sE-cad) is released that has been identified as a pro-carcinogenic inflammatory signal in several bacteria-induced cancers. Recently we reported that $Q$ fever, a disease due to Coxiella burnetii infection, can be complicated by occurrence of non-Hodgkin lymphoma (NHL). Therefore, we studied E-cad switching in $Q$ fever. The sE-cad levels were found increased in the sera of acute and persistent $Q$ fever patients, whereas they remained at the baseline in controls groups of healthy donors, people cured of $\mathrm{Q}$ fever, patients suffering from unrelated inflammatory diseases, and past $\mathrm{Q}$ fever patients who developed NHL. These results indicate that sE-cad can be considered as a new biomarker of $C$. burnetii infection rather than a marker of $\mathrm{NHL}$-associated to $\mathrm{Q}$ fever. We wondered if changes in $\mathrm{SE}$-cad reflected variations in the $\mathrm{CDH} 1$ gene transcription. The expression of E-cad mRNA and its intracellular ligand $\beta$-catenin was down-regulated in peripheral blood mononuclear cells (PBMCs) of patients with either acute or persistent forms of $Q$ fever. Indeed, a lower cell-surface expression of E-cad was measured in a minority $(<5 \%)$ subpopulation of $\mathrm{HLADR}^{+} / \mathrm{CD} 16^{+}$monocytes from patients with acute $Q$ fever. However, a very strong increase in E-cad expression was observed on more than $30 \%$ of the $\mathrm{HLADR}^{+} / \mathrm{CD} 16^{+}$monocytes of persistent $\mathrm{Q}$ fever patients, a cell subpopulation known to be a target for $C$. burnetii in humans. An experimental in vitro infection of healthy donors' PBMCs with $C$. burnetii, was performed to directly evaluate the link between $C$. burnetii interaction with PBMCs and their E-cad expression. A significant increase in the percentage of $\mathrm{HLADR}^{+} / \mathrm{CD}^{+} 6^{+}$monocytes expressing E-cad was measured after PBMCs had been incubated for $8 \mathrm{~h}$ with C. burnetii Nine Mile strain. Altogether, these data demonstrate that $C$. burnetii severely impairs the E-cad expression in circulating cells of $Q$ fever patients.

Keywords: $Q$ fever, coxiella burnetii, adhesion molecule, soluble E-cadherin, biomarker 


\section{INTRODUCTION}

Q fever is a zoonosis most of the time transmitted to humans by animal products contaminated with Coxiella burnetii, a strict intracellular Gram-negative bacterium (Maurin and Raoult, 1999). The bacteria usually infect human by the aerosol route and Q fever usually occurs 2-6 weeks after exposure (van Schaik et al., 2013). In humans, the infection most often remains asymptomatic. Symptomatic infections $(10-60 \%$ of cases), usually resolve spontaneously in a few weeks. This clinical form of the disease, called acute $\mathrm{Q}$ fever, is characterized by high fever, headache, myalgia, pneumonia, and hepatitis (Eldin et al., 2017). In less than 5\% of cases, the symptoms do not resolve and settle in a persistent way (Melenotte et al., 2018). The patients suffering from Q fever can therefore be stratified into different categories ranging from acute $\mathrm{Q}$ fever to persistent $\mathrm{Q}$ fever with or without lymphadenitis in which the bacterium is detectable (Tattevin et al., 2003; Melenotte et al., 2016). The infection can evolve toward endocarditis, vascular infection, osteoarticular infection (Million et al., 2013; Melenotte et al., 2018). Other disorders were also described in association with persistent focalized C. burnetii infections, including interstitial lung diseases, persistent granulomatous hepatitis, and B-cell lymphoma (Ramos et al., 2012; Melenotte et al., 2016, 2018).

So far, the nature of factors that determine the severity of Q fever remain mostly unknown (Deng et al., 2012; Shipman et al., 2013; Stead et al., 2013; Martinez et al., 2014; Schoffelen et al., 2017). Recently, we have reported that the presence of circulating anticardiolipin antibodies during the acute phase of the disease was associated with acute Q fever complications, such as endocarditis, cholecystic, and hemophagocytic syndrome (Melenotte et al., 2018). Until recently, the molecular mechanisms that may account for the progression of Q fever toward lymphoma were largely ignored. Thereby, we investigated the transcriptional signature associated with the development of NHL using the PBMCs of patients with $\mathrm{Q}$ fever. By analyzing their gene expression by microarray, we found the over-expression of genes involved in anti-apoptotic process and repression of pro-apoptotic genes as compared to samples from healthy donors (Melenotte et al., 2019). Yet, the early molecular events controlling the balance between remission and progression toward more severe diseases remain elusive.

There is accumulating evidence reported in the infectious diseases literature indicating that bacterial invasions of human hosts and transmigration at the epithelium level involves the over-expression of sheddases which mediate the degradation of cell surface adhesion molecules (CAM). The cleavage of CAM leads to cellular tissue destruction and rapid spread of bacteria (Elkington et al., 2005; Vanlaere and Libert, 2009). The matrix metalloproteinases (MMP) family, count among the inducible human sheddases that catalyze the proteolysis of cadherins (cad), a group of cell-surface adhesion molecules that includes E-cadherin (E-cad) and belongs to the superfamily of CAM (Grabowska and Day, 2012). Recently, we reported (Jansen et al., 2017) that C. burnetii induces the sheddases MMP-1 and MMP9 production in PBMCs of healthy persons exposed in vitro to the bacterium; that the single nucleotide polymorphisms (SNPs) in MMP-7 and MMP-9 were more frequent in patients with persistent Q fever; and, that the circulating MMP-7 serum levels were higher in patients with persistent $Q$ fever.

The aim of this study was to focus our attention on the modulation of E-cad expression in patients with Q fever. The modulation of E-cad cell-surface expression and the release of a soluble form of E-cad ( $\mathrm{sE}$-cad) have been identified as biomarkers during bacterial invasion of the human host and as a trigger in neoplasic processes induced by several bacterial species. E-cad cleavage has already been associated with breast, gastric and colorectal solid tumors (Repetto et al., 2014), and multiple myeloma induction (Syrigos et al., 2004). It was reported in the literature that Helicobacter pylori, a risk factor for the development of gastric adenocarcinoma, modulates the expression of the E-cad coding gene CDH1 (Jacobs et al., 2011), and activates the calpain sheddase, generating sEcad and inducing a relocalization of $\beta$-catenin $(\beta$-cat), an Ecad intracellular ligand (O'Connor et al., 2011). It was also documented that the Bacteroides fragilis toxin, a known bacterial sheddase, coordinates a pro-carcinogenic inflammatory cascade via the release of $\mathrm{sE}$-cad from colonic epithelial cells, which triggers myeloid cell-dependent distal colon tumorigenesis ( $\mathrm{Wu}$ et al., 2003; Chung et al., 2018). In addition, colorectal tumor development linked to Streptococcus gallolyticus was found to correlate with an increase in $\beta$-cat expression (Kumar et al., 2017). Moreover, phosphorylation of $\beta$-cat by AKT promotes the transcriptional activity of the $\beta$-cat gene (Fang et al., 2007). Finally, increase in $\beta$-cat has been reported in NHL (Ge et al., 2012). Because the cleavage of E-cad and modulation of E-cad/ $\beta$-cat complex expression seem to be a target for several bacteriainducing cancers, we investigated whether a similar mechanism might occur in $\mathrm{Q}$ fever, possibly accounting for shaping a microenvironment favorable to the initiation of NHL.

Herein, we evidenced for the first time that sE-cad is released from cell membrane in patients with $\mathrm{Q}$ fever, and that $C$. burnetii infection modulates the expression of the $\mathrm{CDH}-1 / \mathrm{E}$-cadherin and $C T N N B 1 / \beta$-catenin genes and the cell-surface expression of E-cad on monocytes during $\mathrm{Q}$ fever.

\section{MATERIALS AND METHODS}

\section{Patients Population}

We included 38 patients followed in the French National reference center for Q fever in Marseille France ("Centre National de Référence-CNR des Rickettsia, Coxiella, et bartonella”). Samples were collected from patients with acute $Q$ fever, persistent focalized C. burnetii infection, and persistent focalized C. burnetii lymphadenitis or lymphoma. Acute $\mathrm{Q}$ fever was defined by the association of clinical symptom (fever and/or hepatitis and/or pneumonia and/or lymphadenitis) with serologic criteria for phase II IgG levels $\geq 200$ and phase II IgM $\geq 50$ levels within the 3 months after the onset of symptoms. The persistence of symptoms for more than 3 months in addition to the identification of an infectious focus and to positive serology were the three criteria requested to diagnose persistent focalized C. burnetii infection (Eldin et al., 2017; Melenotte et al., 2018). Clinical characteristics of $Q$ fever patients included are 
summarized in Table 1. It must be emphasized that for reasons of availability of samples that meet the methodological constraints of the assays (i.e., for the sE-cad ELISA, plasma should be collected using heparin as an anticoagulant; EDTA plasma is not suitable for use in this assay due to its chelating properties), it is not systematically the same patients who have been tested by flow cytometry, qRT-PCR, and ELISA. Indeed, samples from 11 patients were tested by two or three assays (samples from nine patients were tested by flow cytometry and qRT-PCR; samples from five patients were tested by flow cytometry and ELISA; samples from three patients were tested using the three different assays). Healthy donors were used as control and samples were obtained from the national blood transfusion center ("Etablissement Français du Sang," EFS, Marseille, France). For the ELISA, in addition to the Q fever patients and healthy donors we also included 10 people cured of Q fever, 5 acute (3 men, 2 women) and 5 persistent (all men) with IgG levels $\geq 800$ (people followed in the French National reference center for Q fever, Marseille, France), and 10 patients (9 women, 1 man) suffering from rheumatoid arthritis (RA) and diagnosed according to the $2010 \mathrm{ACR} /$ EULAR criteria (Aletaha et al., 2010). The mean age of the cured patients was $67 \pm 6.7$ and $49.8 \pm$ 14.9 for persistent and acute Q fever forms, respectively mean \pm standard deviation). The mean age of the RA patient was 60 \pm 12.7 years (mean \pm standard deviation) with disease duration of $15.2 \pm 9.3$ years. Informed written consent was obtained from each subject with the approbation of the ethical committee of the IHU Méditerranée-Infection $\mathrm{n}^{\circ}$ 2016-024.

\section{Cell Isolation}

PBMCs from healthy donors or patients were recovered from samples collected on leukopacks from "Etablissement français du sang" or Ethylenediaminetetraacetate (EDTA) tubes (Sigma Aldrich, Saint-Quentin Fallavier, France), respectively using Ficoll (Eurobio, Les Ulis, France) density gradient centrifugation as previously described (Ka et al., 2016).

\section{Bacteria Production and in vitro Infection of PBMCs}

Coxiella burnetii Nine Mile (RSA496) strain cultured on L929 cells for 8 days were harvested from culture supernatant after cells had been sonicated and centrifuged, as previously described (Ka et al., 2016). C. burnetii present in cell-free supernatants were collected, centrifuged at 10,000 $\mathrm{x}$ for $10 \mathrm{~min}$, washed and stored at $-80^{\circ} \mathrm{C}$ until they were used. For the PBMC infection experiments, $10^{7}$ live bacteria (ratio 1:1) were incubated for $8 \mathrm{~h}$ with $10^{7}$ PBMCs. As controls, PBMCs were either incubated for $8 \mathrm{~h}$ in bacteria-free cell-culture medium or in culture medium supplemented with $10 \mathrm{ng} / \mathrm{ml}$ of lipopolysaccharide (LPS) from Escherichia coli (Sigma Aldrich, Saint-Quentin Fallavier, France).

\section{Ribonucleic Acid (RNA) Isolation}

RNAs were extracted from whole blood or cells using PAXgene blood RNA kit or RNeasy Mini Kit, respectively. This extraction process includes a DNase I treatment to eliminate DNA contaminants according to the manufacturer instructions
TABLE 1 | Patients with C. burnetii infection characteristics.

\begin{tabular}{|c|c|c|c|}
\hline Patient & $\begin{array}{l}\text { Flow cytometry } \\
\qquad n=10\end{array}$ & $\begin{array}{c}\text { q-RTPCR } \\
n=29\end{array}$ & $\begin{array}{l}\text { ELISA } \\
n=26\end{array}$ \\
\hline Sex Men/Women & $8 / 2$ & $21 / 8$ & $22 / 4$ \\
\hline Mean age $( \pm S D)$ & $64.1 \pm 12.6$ & $56 \pm 14.5$ & $54.1 \pm 20.7$ \\
\hline \multicolumn{4}{|l|}{ Acute $Q$ fever } \\
\hline$n=$ & 6 & 14 & 11 \\
\hline Pneumonia only & 2 & 0 & 4 \\
\hline Hepatitis only & 3 & 12 & 7 \\
\hline Pneumonia and hepatitis & 1 & 2 & 0 \\
\hline \multicolumn{4}{|c|}{ Persistent C. burnetii infection } \\
\hline$n=$ & 4 & 15 & 15 \\
\hline Endocarditis & 4 & 14 & 1 \\
\hline Vascular infection & 0 & 1 & 3 \\
\hline Osteoarticular infection & 0 & 0 & 1 \\
\hline Hepatitis only & 0 & 0 & 1 \\
\hline Lymphadenitis & 0 & 0 & 4 \\
\hline Lymphadenitis and hepatitis & 0 & 0 & 3 \\
\hline $\begin{array}{l}\text { Lymphadenitis and } \\
\text { pneumonia }\end{array}$ & 0 & 0 & 0 \\
\hline $\begin{array}{l}\text { Lymphadenitis and } \\
\text { lymphoma }\end{array}$ & 0 & 0 & 2 \\
\hline \multicolumn{4}{|l|}{ Treatment DP } \\
\hline Before treatment & 10 & 4 & 14 \\
\hline During treatment & 0 & 13 & 12 \\
\hline After treatment & 0 & 12 & 10 \\
\hline
\end{tabular}

Bold values represent the total patients included for each groups.

(Qiagen, Courtaboeuf, France) as previously described (Thuny et al., 2012).

\section{Quantitative-Reverse Transcription-Polymerase Chain Reaction (qRT-PCR)}

qRT-PCR was performed to obtain cDNA using the Moloney murine leukemia virus-reverse transcriptase (MMLV-RT) kit (Life Technologies) as previously described (Belhareth et al., 2018). qRT-PCR was realized using the SYBR Green Fast Master Mix (Roche Diagnostics, Meylan, France) and specific primers (designed using the Primer3 software).We used primers for $\mathrm{CDH} 1$ gene (forward: gaaggtgacagagcctctggat and reverse: gatcggttaccgtgatcaaaat) coding for the E-cad mRNA; CTNNB1 gene (forward: agcttccagacacgctatcat and reverse: cggtacaacgagctgtttctac) coding for the $\beta$-cat mRNA; and to the housekeeping gene $\beta$-actin (forward: aggaaggaaggctggaagag and reverse: ggaaatcgtgcgtgacatta). The qRT-PCR results obtained using the $C D H 1$ and $C T N N B 1$ probes were normalized to $\beta$-actin. Data were expressed as $\log 10\left(2^{\Delta \mathrm{Ct}}\right)$, where $\Delta \mathrm{Ct}=$ $\left(\mathrm{Ct}_{\text {Target }}-\mathrm{Ct}_{\text {Actin }}\right)$. The threshold cycle $(\mathrm{Ct})$ was defined as the number of cycles required to detect the fluorescent signal.

\section{Soluble E-Cadherin Quantification}

Sera from acute Q fever, persistent Q fever, rheumatoid arthritis patients and healthy controls were isolated from dry tubes. The quantity of soluble E-cad protein concentration in the patients' 

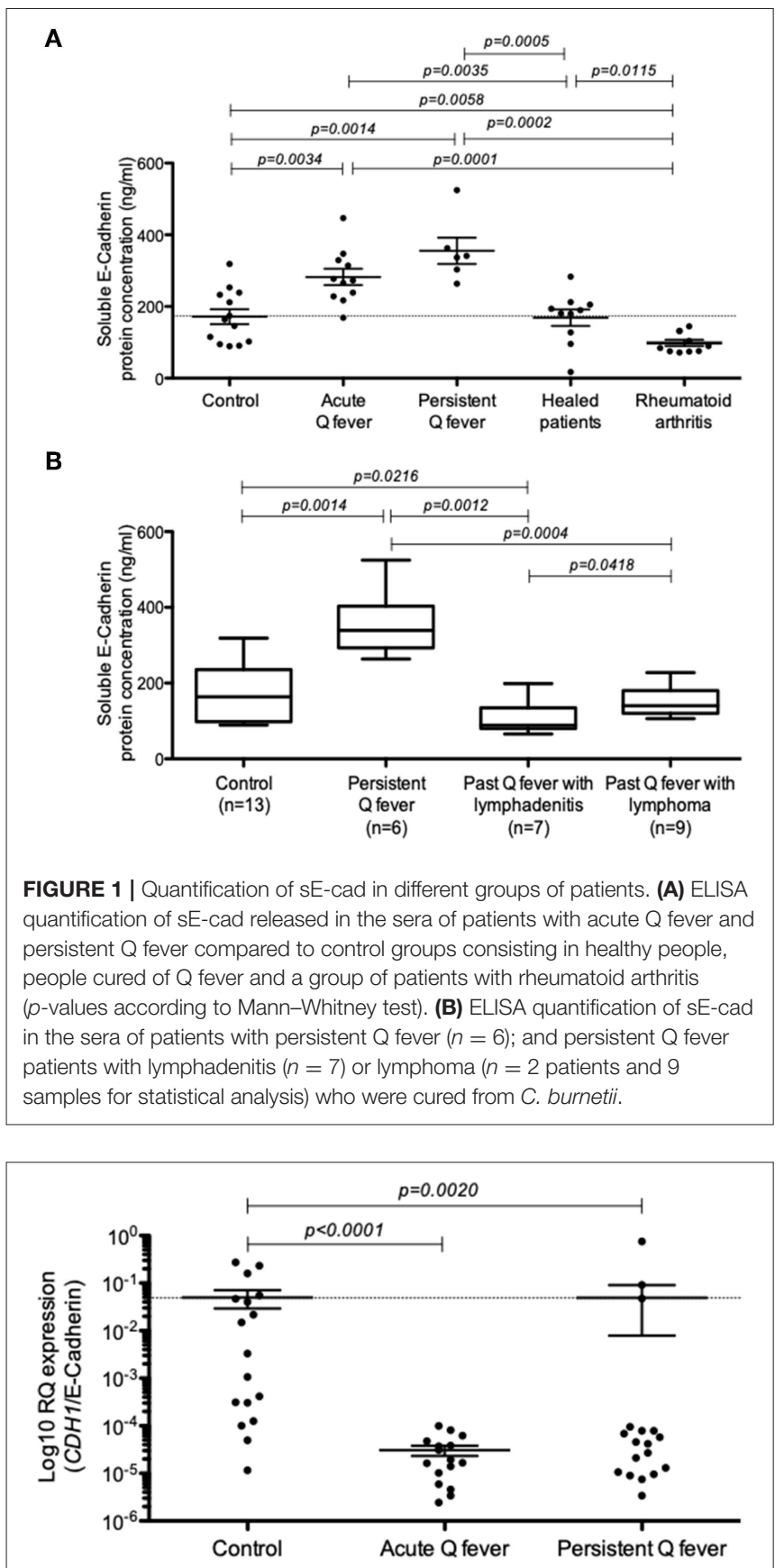

FIGURE 2 | Expression of $C D H 1 /$ E-cadherin in the patients' groups with either acute $Q$ fever $(n=15)$ or persistent $Q$ fever $(n=17)$. mRNA expression was compared to a group of healthy persons $(n=17)$. Data are expressed as $\log 10 R Q$ were $R Q=2^{(-\Delta C T)}$

sera was determined using an immunoassay kit according to the manufacturer's instructions (R\&D system, Lille, France). The minimal detectable concentration of human E-cad is $0.039 \mathrm{ng} / \mathrm{ml}$.

\section{Flow Cytometry}

The membrane expression of E-cad on PBMCs was investigated using flow cytometry. Cells were analyzed according to fluorescence intensity for CD3, CD14, CD16, and CD20, markers. MAb anti-CD3-FITC, anti-CD20-PC5, anti-CD16-PE, and anti-CD14-FITC were purchased from Beckman (Beckman coulter, Villepinte, France). Cell surface expression of E-cad was assayed using an anti-E-cad mAb-APC. Fluorescence intensity was measured using a Canto II cytofluorometer (Becton Dickinson, Biosciences, Le Pont de Claix, France), and the results were analyzed using a FlowJo software X.10.0.7.

\section{Statistical Analysis}

A two-by-two comparison of qRT-PCR data was performed using a two-tailed non-parametric Mann-Whitney $U$-test. All tests were 2 -sided and $p<0.05$ was considered significant.

\section{RESULTS}

\section{Increase of Soluble E-Cadherin in the Sera of Patients With Acute and Persistent Q Fever}

Soluble E-cadherin (sE-cad), the product of a proteolytic cleavage of cell membrane-anchored E-cad by sheddases, has been described in a few infectious diseases to trigger cancers. Using ELISA, we investigated here the presence of sE-cad in the sera of $\mathrm{Q}$ fever patients. As shown in Figure 1A, s-E-cad release was significantly increased in patients with acute Q fever $(n=$ $10 ; p=0.0034)$ and persistent $\mathrm{Q}$ fever $(n=6 ; p=0.0014)$ compared to the two control groups, the healthy donors' group $(n=13)$, the people cured of Q fever $(n=10)$, and the group of patients suffering from rheumatoid arthritis $(n=8)$. These results indicated that $\mathrm{sE}$-cad cleavage and release were specifically observed in the two forms of $\mathrm{Q}$ fever and achieved independently of any inflammatory reaction. We next investigated the serum concentration of sE-cad in the few patients of the cohort who were classified as past persistent focalized Q fever patients but, in addition, suffered from lymphadenitis $(n=7)$ or lymphoma $(n=2)$. The patients carrying lymphomas had been cured from C. burnetii for almost 2 and 8 years, respectively. As shown in Figure 1B, the high levels of sE-cad previously found in the sera of acute and persistent Q fever patients were not found for the patients with either lymphadenitis or lymphoma who were considered cured from C. burnetii. Altogether, these data indicate that serum sE-cad is high in patients with $Q$ fever.

\section{Decreased Expression of CDH1/E-Cadherin mRNA in Cells From Q Fever Patients}

Next, we investigated the expression of E-cad mRNA in the PBMCs of both acute and persistent Q fever groups by qRTPCR. As shown in Figure 2, the expression of E-cad mRNA was significantly down-regulated both in the patients' group with acute Q fever $(n=15 ; p<0.0001)$ and the patients' group with persistent $C$. burnetii infection $(n=17 ; p=0.002)$. It is worth noting that the result of E-cad mRNA modulation was more heterogeneous in the persistent Q fever patients' group than in the group of patients with acute $Q$ fever. Indeed, the persistent $\mathrm{Q}$ fever group included a few patients showing a level of E-cad 
mRNA similar to that of the healthy controls. We must also mention a great heterogeneity among the healthy control group in the $C D H 1 / \mathrm{E}$-cadherin gene expression. These results indicated a significantly reduced expression of E-cad mRNA in the PBMCs of acute $\mathrm{Q}$ fever and most of the persistent $\mathrm{Q}$ fever patients.

\section{Modulation of Cell-Surface Expression of E-Cadherin in Q Fever Patients' Cell Subpopulations}

To go further in the understanding of the E-cad expression in $\mathrm{Q}$ fever, we measured by flow-cytometry the expression of E-cad in PBMCs subsets isolated from the blood of patients with either acute $\mathrm{Q}$ fever or persistent $C$. burnetii infection groups (Figure 3). The flow-cytometry analysis was performed with gating windows on the $\mathrm{CD}^{+}$( $\mathrm{T}$ cells), $\mathrm{CD}^{2} 0^{+}$(B-cells), and both $\mathrm{CD}_{14}{ }^{+}$and $\mathrm{CD}^{+} 6^{+}$(monocytes) subpopulations. In the PBMCs from healthy donors, the E-cad was expressed by $<5 \%$ of the $\mathrm{CD}^{+}$lymphocytes, $<1 \%$ of the $\mathrm{CD} 20^{+}$ lymphocytes, and $9-25 \%$ of the HLA-DR ${ }^{+}$monocytes. A shift in fluorescence intensity for E-cad labeling was observed in some cell subpopulations both in samples from acute $(n=$ 7 ) and persistent focalized $\mathrm{Q}$ fever patients $(n=4)$. While E-cad expression at the cell surface of $\mathrm{CD}^{+}$cells remained similar in patients and controls, we found a moderate but significant decrease of E-cad expression that concerns about $3 \%$ of the $\mathrm{CD}^{+} 0^{+}$cells during both acute $(p=0.0012)$ and persistent focalized ( $p=0.0044) \mathrm{Q}$ fever. Although, cell-surface expression of E-cad remained stable in $\mathrm{HLADR}^{+} / \mathrm{CD} 14^{+}$cells, or significantly decreased in $\mathrm{HLADR}^{+} / \mathrm{CD} 6^{+}$cells from acute $\mathrm{Q}$ fever patients $(p=0.0004)$, it was significantly increased in both the HLADR ${ }^{+} / \mathrm{CD} 14^{+}(p=0.0039)$ and $\mathrm{HLADR}^{+} / \mathrm{CD} 6^{+}$ ( $p=0.0039)$ cell subpopulations from persistent $\mathrm{Q}$ fever patients. A greater over-expression of cell surface E-cad was found within more than $30 \%$ of the $\mathrm{HLADR}^{+} / \mathrm{CD} 16^{+}$subpopulation. At the level of cell subpopulations, the reduction of E-cad protein expression in B-cells corroborates the decrease in E-cad mRNA observed with PBMCs of Q fever patients. Despite the significant global decrease in E-cad mRNA observed with the PBMCs of both acute and persistent $Q$ fever patients, we found a surprising over-expression of cell-surface E-cad protein on the $\mathrm{HLADR}^{+} / \mathrm{CD} 6^{+}$cells of persistent $\mathrm{Q}$ fever patients.

\section{Differential Expression of CTNNB1/ $\beta$-Catenin mRNA Between Acute and Persistent $Q$ Fever Patients}

Because E-Cad protein expressed at the cell membrane is expected to bind $\beta$-cat through its intracytoplasmic tail and thus regulate cell signaling, we investigated whether or not $Q$ fever is associated with a modulation of $\beta$-cat mRNA expression. We found that the expression of CTNNB1 mRNA coding for $\beta$-cat in PBMCs of Q fever patients was significantly reduced in acute $\mathrm{Q}$ fever $(n=15 ; p=0.0029)$ compared to healthy controls and patients with persistent $Q$ fever (Figure 4). The $\beta$ cat mRNA modulation was more heterogeneous in the persistent Q fever patients' group $(n=17)$; within this group, there was no statistically significant difference with the healthy control group. These results indicate that $\beta$-cat gene expression is downmodulated during the acute phase of $\mathrm{Q}$ fever, yet its expression may then vary in patients who suffer from persistent $Q$ fever.

\section{Modulation of CDH-1 Gene Expression and Membrane E-cad in PBMCs Exposed in vitro to C. burnetii Infection}

In order to evidence a direct relationship between the modulation of E-cad expression and the presence of $C$. burnetii in the culture medium of human cells, PBMCs from six healthy donors were exposed in vitro during $8 \mathrm{~h}$ to $10^{7}$ live C. burnetii Nine Mile (RSA493) reference strain. The modulation of $C D H 1 / \mathrm{E}-$ cadherin mRNA and $C T N N B 1 / \beta$-catenin mRNA was analyzed by qRT-PCR. As shown in Figure 5A, there was a tendency for over-expression of CDH1/E-cadherin mRNA in PBMCs exposed to $C$. burnetii but it was not statistically significant. A significant ( $p=0.0073$ ) over-expression of $C T N N B 1 / \beta$-catenin mRNA was found for PBMCs exposed to C. burnetii. It is worth noting that the same tendency for over-expression of $C D H 1 /$ E-cadherin mRNA was observed after PBMCs had been exposed to LPS from $E$. coli and a significant ( $p=0.0413)$ overexpression of $C T N N B 1 / \beta$-catenin mRNA was found under these experimental conditions.

In order to determine the cell subpopulation(s) on which $C$. burnetii might act on cellular genes transcription to modulate the expression of E-cad, PBMCs were fixed $8 \mathrm{~h}$ after incubation with C. burnetii Nine Mile strain and analyzed by flow-cytometry for E-cad surface expression. As shown in Figure 5B, a significant increase in the percentage of HLA-DR+/CD16+ monocytes expressing E-cad at their surface was measured. This result corroborates the data got with cell subpopulations isolated from persistent Q fever patients.

\section{DISCUSSION}

We report here the first experimental evidence that $\mathrm{sE}$-cad is detected in the sera of patients with acute or persistent $Q$ fever, indicating that sE-cad can be considered as a new biomarker of $\mathrm{Q}$ fever. The release of sE-cad is accompanied by modifications in the transcription of the $C D H 1$ gene and by modulation of the E-cad expression at the surface of cells.

Increased serum levels of sE-cad have been reported in a number of metabolic and inflammatory diseases as well as cancers (Grabowska and Day, 2012). Moreover, a role for sEcad as tumorogenic co-factor was highlighted in the infections by $H$. pylori, B. fragilis, and S. gallolyticus, that trigger gastric adenocarcinoma or colorectal tumors (Wu et al., 2003; O'Connor et al., 2011; Kumar et al., 2017; Chung et al., 2018). Herein, we demonstrate that sE-cad is increased in Q fever but fail to find an association between sE-cad release and the induction of NHL. Our data strongly suggest that the presence of sE-cad in the sera of $\mathrm{Q}$ fever patients is a marker of a metabolic disorder and/or bacterial invasion. Measurement of sE-cad could advantageously be added to the set of biological parameters aimed at monitoring patients with $\mathrm{Q}$ fever. Interestingly, sE-cad is no longer found increased in the sera of patients who had been considered 
A
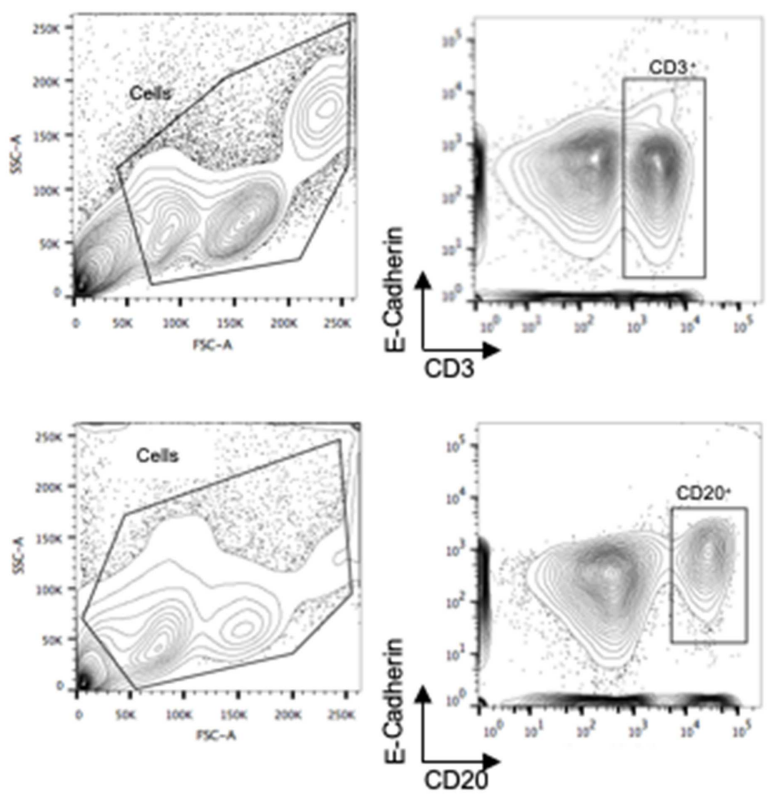

B
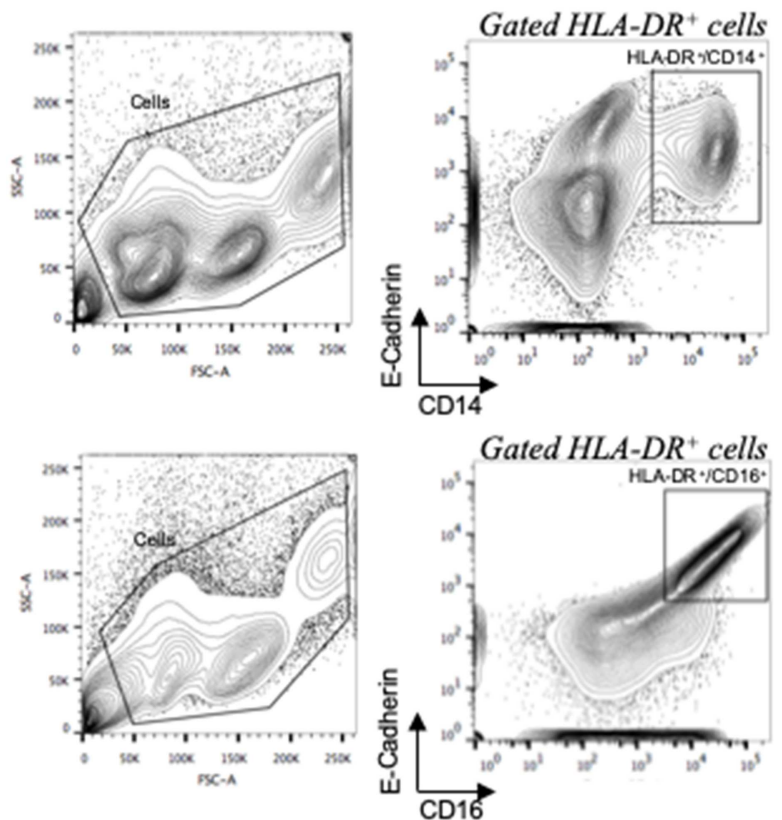

\section{E-cadherin ${ }^{+}$}

cells (\%)
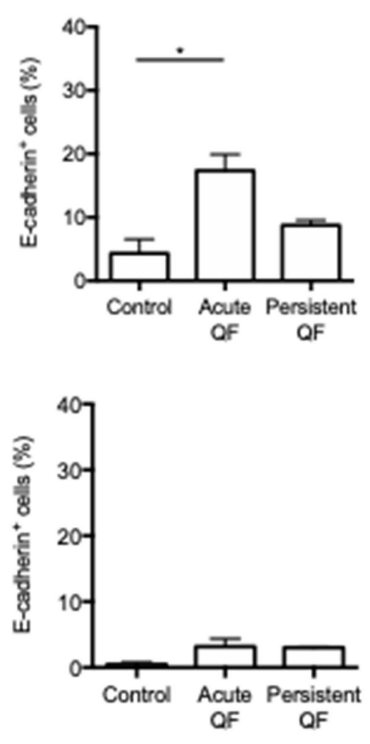

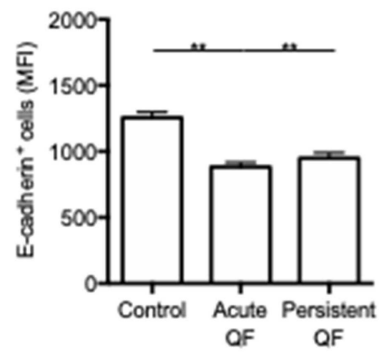

\section{E-cadherin ${ }^{+}$ \\ cells (MFI)}

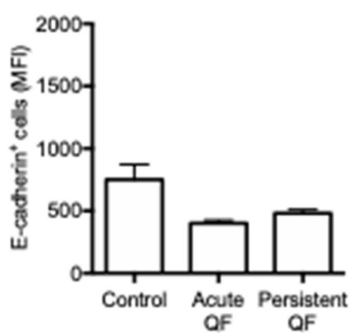

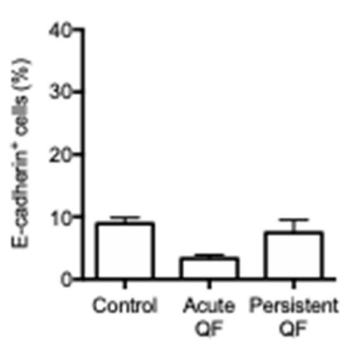
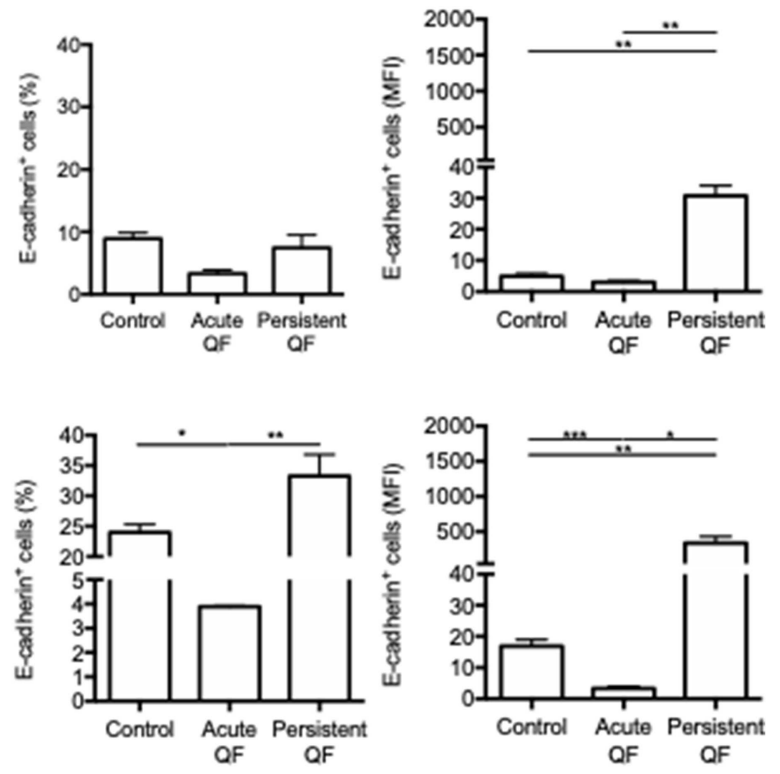

FIGURE 3 | Flow cytometry analysis of E-cadherin expression at the surface of cells from patients with acute $Q$ fever $(n=7)$ and persistent $Q$ fever $(n=4)$ compared to samples from healthy persons $(n=13)$. The gating was performed using different cluster differentiation-specific mAb allowing to select subpopulations of lymphocytes (A) $\mathrm{CD}^{+}$and $\mathrm{CD}_{2} 0^{+}$; (B) monocytes $\mathrm{HLADR}{ }^{+} / \mathrm{CD} 14^{+}$and $\mathrm{HLADR}^{+} / \mathrm{CD}_{16}{ }^{+}$. The left panels define the gating chosen; the middle panels show the percent of cells expressing E-cadherin with respect to the cell subpopulations analyzed and the patient's group (healthy donor = control; acute fever patients = Acute QF; and persistent Q fever patients = Persistent QF); the right panels are the histograms of E-cad cell surface expression (MFI) with respect to the cell subpopulations analyzed and the patient's group. 


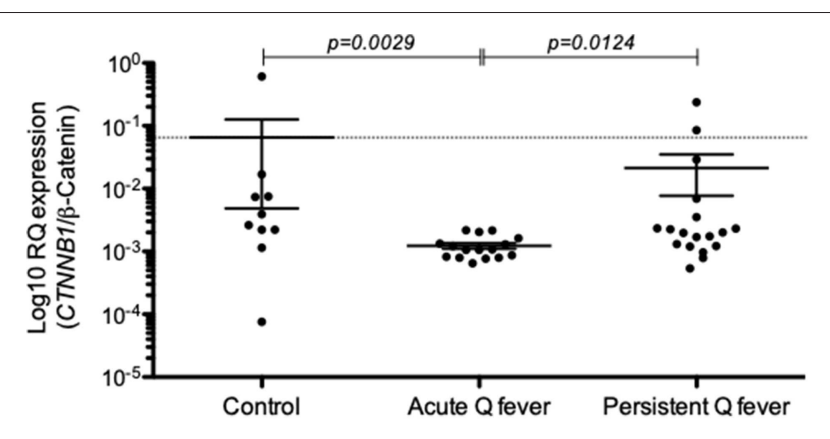

FIGURE 4 | Expression of CTNNB1/ $\beta$-catenin mRNAs in the patients' groups with either acute $Q$ fever $(n=15)$ or persistent $Q$ fever $(n=17)$. mRNA expression was compared to a group of healthy persons $(n=10)$. Data are expressed as $\log 10 R Q$ were $R Q=2^{(-\Delta C T)}$

cured of C. burnetii (people who lack clinical symptoms but show elevated phase I IgG). These observations suggest that sE-cad release might require the presence of $C$. burnetiiinfected cells circulating in the patient. Working on patients' cell subpopulations, we found a significant decreased of E-cad that concerns a small percentage $(<5 \%)$ of the CD20 ${ }^{+}$B-cells subpopulation. In the in vitro infection assay, we also observed the same tendency for the $\mathrm{CD} 20^{+} \mathrm{B}$-cells. This E-cad downregulation could either be due to sheddase activation or $\mathrm{CDH} 1$ gene repression in $\mathrm{B}$-cell infected by $C$. burnetii, continuous exposure of B cells to C. burnetii lipopolysaccharides, or aberrant bystander cell signaling in their microenvironment (Amano et al., 1987; Lee, 1993; Melenotte et al., 2016). Heterotypic binding of E-cad on epithelial tissues to CD103 on lymphocytes is welldocumented. In contrast the functional role of E-cad on the surface of lymphocytes has not yet been addressed in vivo. Although the expression of E-cad on subpopulations of mature human lymphocytes has not been the subject of intense research until now because this molecule is usually absent from mature lymphocytes, it is likely that the differential expression of these molecules under pathological conditions reflects changes in the transmigration and homing capacity of these cells (Reyat et al., 2017). Conversely, we found that the E-cad protein expression at the surface of the cells of patients with $Q$ fever was significantly increased on both $\mathrm{CD}_{1} 4^{+}$and $\mathrm{CD}_{16}{ }^{+}$monocytes from persistent $\mathrm{Q}$ fever patients. We observed a greater expression of E-cad that concerns more than $30 \%$ of $\mathrm{CD} 16^{+}$monocytes, a cell subpopulation that we previously demonstrated as being a target for C. burnetii in humans (Ka et al., 2016). In addition, we found that $C$. burnetii infection triggers a reduced expression of E-cad mRNA in the PBMCs of acute Q fever patients. Although sE-cad release in sera and decreased E-cad mRNA expression in PBMCs was also observed in the persistent Q fever patients' group, it is clear that there are marked differences between individuals within this second group, and their mean level of E-cad mRNA was found similar to that of the healthy controls. Moreover, in contrast to the data obtained with cell subpopulations of acute Q fever patients, high cell surface expression of E-cad was found in both $\mathrm{CD} 14^{+}$and $\mathrm{CD}_{1} 6^{+}$cell subpopulations from persistent Q fever patients. When PBMCs from healthy donors were exposed in vitro to C. burnetii Nine Mile strain for $8 \mathrm{~h}$ and analyzed by flow cytometry, about $30 \%$ of the CD $16+$ monocytes were found to express cell-surface E-cad. This increase in the percentage of cells expressing cell-surface E-cad might be either due to $C$. burnetii polysaccharide induced signaling after cellsurface binding or transduction of signal by intracellular live bacteria. In vitro infection studies by Heinzen and collaborators have shown that the avirulent strain of C. burnetii induced DCs maturation, whereas cell maturation was not observed with the Nine Mile virulent strain of C. burnetii (Shannon et al., 2005; Larson and Heinzen, 2017).

Although rare, the incidence of NHL B-lymphoma in patients infected by C. burnetii was significantly higher (25fold) than within the general French population (Melenotte et al., 2016). Despite intensive work to identify the bacterial compounds supporting C. burnetii replication and virulence (Deng et al., 2012; Shipman et al., 2013; Stead et al., 2013; Martinez et al., 2014, 2016), the molecular mechanism involved in C. burnetii-induced Q fever and NHL-associated to Q fever remains largely unknown. Interestingly, E-cad is known to act as a tumor-suppressing protein which inhibits the canonical Wnt-Frizzled signaling pathway by sequestration of $\beta$-Cat. Besides, the aberrant expression of E-cad can contribute to cellular transformation through $\beta$-Cat dysregulation leading to activation of $\beta$-Cat /T-cell factor signaling (Gottardi et al., 2001; Marambaud, 2002; Gall and Frampton, 2013; Kourtidis et al., 2013). The shedding of sE-cad can also allow extracellular domains of E-cad interaction with the ErbB receptors inducing their transactivation (Zheng et al., 2009). Therefore, we speculate that the release of $\mathrm{sE}$-cad might participate to the molecular crosstalk which takes place in the microenvironment of the lymph node during persistent $\mathrm{Q}$ fever and might possibly trigger a pro-carcinogenic program required for the initiation of NHL lymphoma. In this hypothesis, one should expect that E-cad cleavage would allow $\beta$-cat translocation to the nucleus for trans-activation of proliferative genes. Surprisingly, we observed a down-regulation in $\beta$-cat mRNA expression in PBMCs of acute and persistent $\mathrm{Q}$ fever patients, which does not seem support this model and remains to be explained. Yet, when PBMCs were exposed in vitro to $C$. burnetii, a significant over-expression of $C T N N B 1 / \beta$-catenin mRNA was measured, and this phenomenon should be further explored. In $\mathrm{Q}$ fever, overproduction of interleukin-10 (IL-10) by infected monocytes was found critical in both sustaining replication of the C. burnetii and preventing the macrophages microbicidal activity (Mege et al., 2006; Ka et al., 2014). High levels of this B-cell growth factor are known to increase BCL-2 expression in lymphoma cells (Voorzanger et al., 1996), and to correlate with a worse prognosis in DLBCL patients (Lech-Maranda et al., 2004). The Rituximab used in the treatment of NHL patients may overcome BCL-2-mediated chemoresistance through the inhibition of IL-10, thus downregulating BCL-2 expression (Park et al., 2009). By investigating a specific gene signature for $\mathrm{Q}$ fever patients by microarray, we recently found that specific genes involved in anti-apoptotic process were over-expressed compared to samples from healthy donors, and that pro-apoptotic genes were repressed (Melenotte et al., 2019). 
A
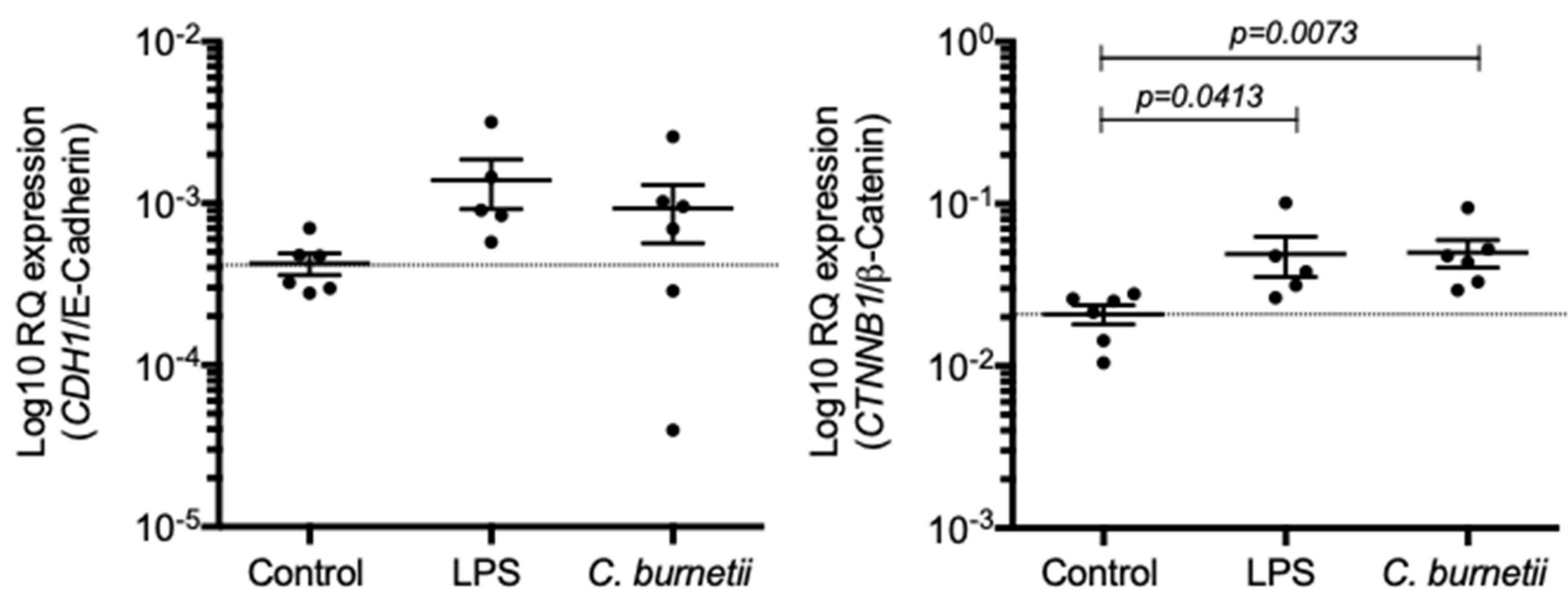

B
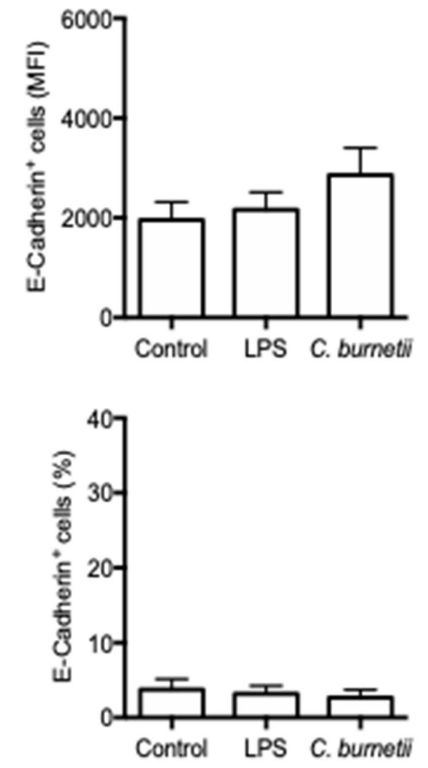

Gated $\mathrm{CD}^{2} \mathrm{O}^{+}$cells
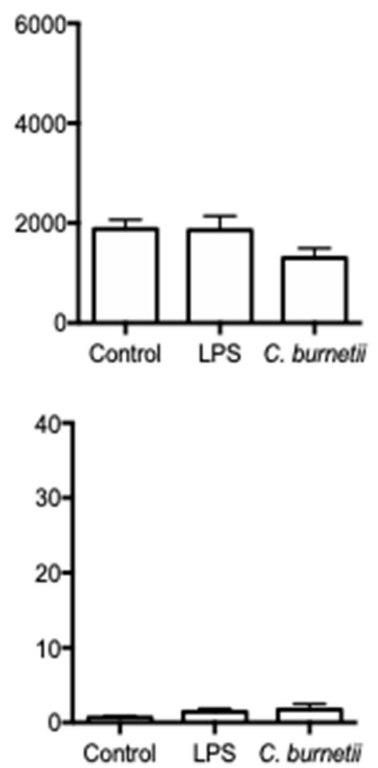

Gated $H L A-D R^{+} / C D 14^{+}$cells
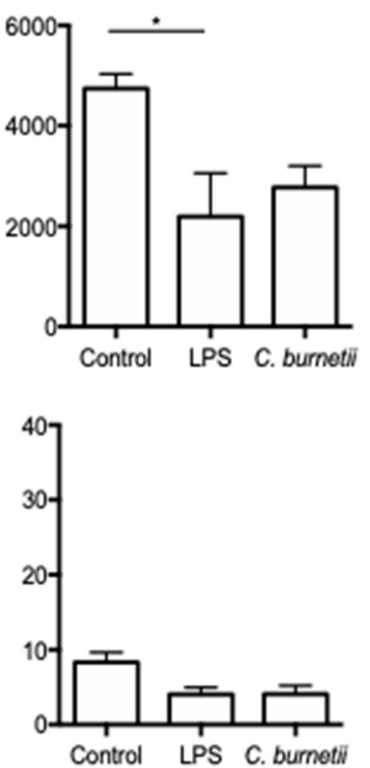

Gated $H L A-D R^{+} / C D 16^{+}$cells
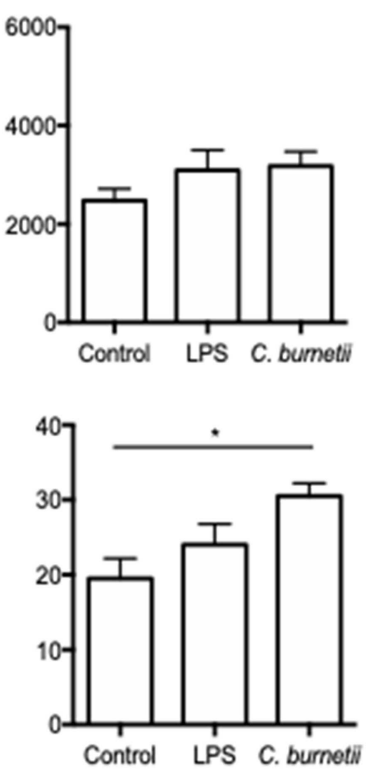

FIGURE 5 | (A) qRT-PCR analysis of CDH1/E-cadherin (left panel) and CTNNB1/ $\beta$-catenin (right panel) mRNAs expression in PBMCs from six healthy donors exposed in vitro during $8 \mathrm{~h}$ to $\mathrm{C}$. burnetii Nine Mile strain. The gene expression is compared to that of PBMCs maintained germ-free (control) and PBMCs cultured in medium supplemented with LPS from E. coli. (B) Flow cytometry analysis of E-cad expression at the surface of PBMCs (from six different donors) exposed in vitro for $8 \mathrm{~h}$ to live $\mathrm{C}$. burnetii. The gating was performed using different cluster differentiation-specific mAb allowing to select subpopulations of lymphocytes: $\mathrm{CD}^{+}{ }^{+} \mathrm{CD}^{2} \mathrm{O}^{+}$, and monocytes $\mathrm{HLADR}^{+} / \mathrm{CD}_{14}{ }^{+}$and $\mathrm{HLADR}^{+} / \mathrm{CD}_{16}{ }^{+}$.

Another interesting question concerns the molecular mechanism by which cleavage of E-cad is achieved (Niessen et al., 2011). It is likely that E-cad underwent a catalytic cleavage by a protease of human origin during the acute and persistent phases of the disease. Yet, we cannot currently exclude that a sheddase of bacterial origin could be produced by C. burnetii. In the literature the proteolytic cleavage of E-cad was reported for a number of sheddases, including zinc-dependent matrix metalloproteinases (MMP-2, -3, -7, -9 and -14), members of the disintegrin family (adamalysin/ADAM-10 and-15), bacterial proteases gingipains (HRgpA, RgpB, and Kgp), $B$. fragilis toxin/fragilysin, cysteine cathepsins (B, L, and S), serine protease Kallikrein-7 (KLK7), plasmin serine protease, aspartic proteinases BACE1 and BACE2, and malaria parasite serine proteinases PfSUB2n, PfROM1, and PfROM 4 (Grabowska and Day, 2012). One possible candidate for E-cad cleavage during C. burnetii infections could be the human MMP-9, since: (i) MMP-9 levels were reported elevated in the sera of patients with 
endocarditis (Thuny et al., 2012), and patients with acute Q fever (Krajinović et al., 2012); (ii) its production was induced in PBMCs of healthy persons following in vitro exposure to $C$. burnetii; (iii) MMP-9 SNP was found more frequently in patients with persistent Q fever (Jansen et al., 2017); and (iv) MMP-9 was recently identified as a key gene in mantle cell lymphoma (Yan et al., 2018). Experiments are under progress in our laboratory to investigate whether $C$. burnetii infection induces the activation of a human cellular protease from the sheddase family or if $C$. burnetii itself encodes for a sheddase.

In conclusion, this work describes for the first time the modulation of the E-cad signaling pathway and release of sE-cad in the sera of Q fever patients. It opens a new avenue of research to understand the pathophysiology of $\mathrm{Q}$ fever and the role played by E-cad during C. burnetii invasion of human tissues.

\section{DATA AVAILABILITY}

The raw data supporting the conclusions of this manuscript will be made available by the authors, without undue reservation, to any qualified researcher.

\section{REFERENCES}

Aletaha, D., Neogi, T., Silman, A. J., Funovits, J., Felson, D. T., Bingham, C. O., et al. (2010). 2010 Rheumatoid arthritis classification criteria: an American College of Rheumatology/European League Against Rheumatism collaborative initiative. Arthritis Rheum. 62, 2569-2581. doi: 10.1002/art.27584

Amano, K., Williams, J. C., Missler, S. R., and Reinhold, V. N. (1987). Structure and biological relationships of Coxiella burnetii lipopolysaccharides. J. Biol. Chem. 262, 4740-4747.

Belhareth, R., Mezouar, S., Ben Amara, A., Chartier, C., Azzouz, E. B., Chabrière, E., et al. (2018). Cigarette smoke extract interferes with placenta macrophage functions: a new mechanism to compromise placenta functions? Reprod. Toxicol. 78, 120-129. doi: 10.1016/j.reprotox.2018.04.009

Chung, L., Orberg, E. T., Geis, A. L., Chan, J. L., Fu, K., DeStefano Shields, C. E., et al. (2018). Bacteroides fragilis toxin coordinates a pro-carcinogenic inflammatory cascade via targeting of colonic epithelial cells. Cell Host Microbe 23, 203-214.e5. doi: 10.1016/j.chom.2018.01.007

Deng, W., Yu, H. B., de Hoog, C. L., Stoynov, N., Li, Y., Foster, L. J., et al. (2012). Quantitative proteomic analysis of type III secretome of enteropathogenic Escherichia coli reveals an expanded effector repertoire for attaching/effacing bacterial pathogens. Mol. Cell. Proteomics 11, 692-709. doi: $10.1074 / \mathrm{mcp} . M 111.013672$

Eldin, C., Mélenotte, C., Mediannikov, O., Ghigo, E., Million, M., Edouard, S., et al. (2017). From Q fever to coxiella burnetii infection: a paradigm change. Clin. Microbiol. Rev. 30, 115-190. doi: 10.1128/CMR.00045-16

Elkington, P. T. G., O'Kane, C. M., and Friedland, J. S. (2005). The paradox of matrix metalloproteinases in infectious disease. Clin. Exp. Immunol. 142, 12-20. doi: 10.1111/j.1365-2249.2005.02840.x

Fang, D., Hawke, D., Zheng, Y., Xia, Y., Meisenhelder, J., Nika, H., et al. (2007). Phosphorylation of beta-catenin by AKT promotes beta-catenin transcriptional activity. J. Biol. Chem. 282, 11221-11229. doi: 10.1074/jbc.M611871200

Gall, T. M. H., and Frampton, A. E. (2013). Gene of the month: E-cadherin (CDH1). J. Clin. Pathol. 66, 928-932. doi: 10.1136/jclinpath-2013-201768

Ge, X., Lv, X., Feng, L., Liu, X., and Wang, X. (2012). High expression and nuclear localization of $\beta$-catenin in diffuse large B-cell lymphoma. Mol. Med. Rep. 5, 1433-1437. doi: $10.3892 / \mathrm{mmr} .2012 .835$

Gottardi, C. J., Wong, E., and Gumbiner, B. M. (2001). E-cadherin suppresses cellular transformation by inhibiting beta-catenin signaling in an adhesionindependent manner. J. Cell Biol. 153, 1049-1060.

\section{AUTHOR CONTRIBUTIONS}

SM, IO, and CS performed the experiments. CS and CM analyzed clinical data. SM and CC performed the flow cytometry analysis. CD supervised the experimental work. J-LM and DR supervised the clinical studies. SM, J-LM, and CD conceived and wrote the paper.

\section{ACKNOWLEDGMENTS}

SM was first supported by a Fondation pour la Recherche Médicale postdoctoral fellowship (reference: SPF20151234951) and then by the Fondation Méditerranée Infection. We acknowledge Professor Daniel Olive for his kind donation of anti-E-cadherin $\mathrm{mAb}$ and Daniel Bertin for providing sera from rheumatoid arthritis patients. We thank Magdalen Lardière for English editing. This work was supported by the French Government under the Investissements d'avenir (Investments for the Future) program managed by the Agence Nationale de la Recherche (reference: Méditerranée Infection 10-IAHU-03).

Grabowska, M. M., and Day, M. L. (2012). Soluble E-cadherin: more than a symptom of disease. Front. Biosci. Landmark Ed. 17, 1948-1964.

Jacobs, G., Hellmig, S., Huse, K., Titz, A., Franke, A., Kwiatkowski, R., et al. (2011). Polymorphisms in the 3'-untranslated region of the $\mathrm{CDH} 1$ gene are a risk factor for primary gastric diffuse large B-cell lymphoma. Haematologica 96, 987-995. doi: 10.3324 /haematol.2010.033126

Jansen, A. F. M., Schoffelen, T., Textoris, J., Mege, J. L., Bleeker-Rovers, C. P., Roest, H. I. J., et al. (2017). Involvement of matrix metalloproteinases in chronic Q fever. Clin. Microbiol. Infect. Off. Publ. Eur. Soc. Clin. Microbiol. Infect. Dis. 23, 487.e7-487.e13. doi: 10.1016/j.cmi.2017.01.022

Ka, M. B., Gondois-Rey, F., Capo, C., Textoris, J., Million, M., Raoult, D., et al. (2014). Imbalance of circulating monocyte subsets and PD-1 dysregulation in Q fever endocarditis: the role of IL-10 in PD-1 modulation. PLoS ONE 9:e107533. doi: 10.1371/journal.pone.0107533

Ka, M. B., Mezouar, S., Ben Amara, A., Raoult, D., Ghigo, E., Olive, D., et al. (2016). Coxiella burnetii induces inflammatory interferon-like signature in plasmacytoid dendritic cells: a new feature of immune response in Q fever. Front. Cell. Infect. Microbiol. 6:70. doi: 10.3389/fcimb.2016.00070

Kourtidis, A., Ngok, S. P., and Anastasiadis, P. Z. (2013). p120 catenin: an essential regulator of cadherin stability, adhesion-induced signaling, and cancer progression. Prog. Mol. Biol. Transl. Sci. 116, 409-432. doi: 10.1016/B978-0-12-394311-8.00018-2

Krajinović, L. C., Soprek, S., Korva, M., DŽelalija, B., Rode, O. Ã., Skerk, V., et al. (2012). Serum levels of metalloproteinases and their inhibitors during infection with pathogens having integrin receptor-mediated cellular entry. Scand. J. Infect. Dis. 44, 663-669. doi: 10.3109/00365548.2012. 677060

Kumar, R., Herold, J. L., Schady, D., Davis, J., Kopetz, S., MartinezMoczygemba, M., et al. (2017). Streptococcus gallolyticus subsp. gallolyticus promotes colorectal tumor development. PLoS Pathog. 13:e1006440. doi: 10.1371/journal.ppat.1006440

Larson, C. L., and Heinzen, R. A. (2017). High-Content imaging reveals expansion of the endosomal compartment during Coxiella burnetii parasitophorous vacuole maturation. Front. Cell. Infect. Microbiol. 7:48. doi: 10.3389/fcimb. 2017.00048

Lech-Maranda, E., Baseggio, L., Bienvenu, J., Charlot, C., Berger, F., Rigal, D., et al. (2004). Interleukin-10 gene promoter polymorphisms influence the clinical outcome of diffuse large B-cell lymphoma. Blood 103, 3529-3534. doi: 10.1182/blood-2003-06-1850 
Lee, W. Y. (1993). Hairy cell transformation of human peripheral blood lymphocytes by Coxiella burnetii. Yonsei Med. J. 34, 11-21. doi: 10.3349/ymj.1993.34.1.11

Marambaud, P. (2002). A presenilin-1/gamma-secretase cleavage releases the Ecadherin intracellular domain and regulates disassembly of adherens junctions. EMBO J. 21, 1948-1956. doi: 10.1093/emboj/21.8.1948

Martinez, E., Allombert, J., Cantet, F., Lakhani, A., Yandrapalli, N., Neyret, A., et al. (2016). Coxiella burnetii effector CvpB modulates phosphoinositide metabolism for optimal vacuole development. Proc. Natl. Acad. Sci. U.S.A. 113, E3260-E3269. doi: 10.1073/pnas.1522811113

Martinez, E., Cantet, F., Fava, L., Norville, I., and Bonazzi, M. (2014). Identification of OmpA, a Coxiella burnetii protein involved in host cell invasion, by multi-phenotypic high-content screening. PLoS Pathog. 10:e1004013. doi: 10.1371/journal.ppat.1004013

Maurin, M., and Raoult, D. (1999). Q fever. Clin. Microbiol. Rev. 12, 518-553.

Mege, J. L., Meghari, S., Honstettre, A., Capo, C., and Raoult, D. (2006). The two faces of interleukin 10 in human infectious diseases. Lancet Infect. Dis. 6, 557-569. doi: 10.1016/S1473-3099(06)70577-1

Melenotte, C., Mezouar, S., Ben Amara, A., Benatti, S., Chiaroni, J., Devaux, C., et al. (2019). A transcriptional signature associated with non-Hodgkin lymphoma in the blood of patients with Q fever. PLoS One 14:e0217542. doi: 10.1371/journal.pone. 0217542

Melenotte, C., Million, M., Audoly, G., Gorse, A., Dutronc, H., Roland, G., et al. (2016). B-cell non-Hodgkin lymphoma linked to Coxiella burnetii. Blood 127, 113-121. doi: 10.1182/blood-2015-04-639617

Melenotte, C., Protopopescu, C., Million, M., Edouard, S., Carrieri, M. P., Eldin, C., et al. (2018). Clinical features and complications of Coxiella burnetii infections from the french national reference center for Q fever. JAMA Netw. Open 1:e181580. doi: 10.1001/jamanetworkopen.2018.1580

Million, M., Walter, G., Thuny, F., Habib, G., and Raoult, D. (2013). Evolution from acute $Q$ fever to endocarditis is associated with underlying valvulopathy and age and can be prevented by prolonged antibiotic treatment. Clin. Infect. Dis. Off. Publ. Infect. Dis. Soc. Am. 57, 836-844. doi: 10.1093/cid/cit419

Niessen, C. M., Leckband, D., and Yap, A. S. (2011). Tissue organization by cadherin adhesion molecules: dynamic molecular and cellular mechanisms of morphogenetic regulation. Physiol. Rev. 91, 691-731. doi: 10.1152/physrev.00004.2010

O'Connor, P. M., Lapointe, T. K., Jackson, S., Beck, P. L., Jones, N. L., and Buret, A. G. (2011). Helicobacter pylori activates calpain via toll-like receptor 2 to disrupt adherens junctions in human gastric epithelial cells. Infect. Immun. 79, 3887-3894. doi: 10.1128/IAI.05109-11

Park, Y. H., Sohn, S. K., Kim, J. G., Lee, M. H., Song, H. S., Kim, M. K., et al. (2009). Interaction between BCL2 and interleukin-10 gene polymorphisms alter outcomes of diffuse large B-cell lymphoma following rituximab plus CHOP chemotherapy. Clin. Cancer Res. Off. J. Am. Assoc. Cancer Res. 15, 2107-2115. doi: 10.1158/1078-0432.CCR-08-1588

Ramos, J. C., Sin, S. H., Staudt, M. R., Roy, D., Vahrson, W., Dezube, B. J., et al. (2012). Nuclear factor kappa B pathway associated biomarkers in AIDS defining malignancies. Int. J. Cancer 130, 2728-2733. doi: 10.1002/ijc.26302

Repetto, O., De Paoli, P., De Re, V., Canzonieri, V., and Cannizzaro, R. (2014). Levels of soluble E-cadherin in breast, gastric, and colorectal cancers. BioMed Res. Int. 2014:408047. doi: 10.1155/2014/408047

Reyat, J. S., Chimen, M., Noy, P. J., Szyroka, J., Rainger, G. E., and Tomlinson, M. G. (2017). ADAM10-interacting tetraspanins Tspan 5 and Tspan 17 regulate VEcadherin expression and promote $\mathrm{T}$ lymphocyte transmigration. J. Immunol. Baltim. Md 199, 666-676. doi: 10.4049/jimmunol.1600713

Schoffelen, T., Textoris, J., Bleeker-Rovers, C. P., Ben Amara, A., van der Meer, J. W. M., Netea, M. G., et al. (2017). Intact interferon- $\gamma$ response against Coxiella burnetii by peripheral blood mononuclear cells in chronic $\mathrm{Q}$ fever.
Clin. Microbiol. Infect. Off. Publ. Eur. Soc. Clin. Microbiol. Infect. Dis. 23, 209.e9-209.e15. doi: 10.1016/j.cmi.2016.11.008

Shannon, J. G., Howe, D., and Heinzen, R. A. (2005). Virulent Coxiella burnetii does not activate human dendritic cells: role of lipopolysaccharide as a shielding molecule. Proc. Natl. Acad. Sci. U.S.A. 102, 8722-8727. doi: 10.1073/pnas.0501863102

Shipman, M., Lubick, K., Fouchard, D., Gurram, R., Grieco, P., Jutila, M., et al. (2013). Proteomic and systems biology analysis of the monocyte response to Coxiella burnetii infection. PLoS ONE 8:e69558. doi: 10.1371/journal.pone.0069558

Stead, C. M., Omsland, A., Beare, P. A., Sandoz, K. M., and Heinzen, R. A. (2013). Sec-mediated secretion by Coxiella burnetii. BMC Microbiol. 13:222. doi: 10.1186/1471-2180-13-222

Syrigos, K. N., Harrington, K. J., Karayiannakis, A. J., Baibas, N., Katirtzoglou, N., and Roussou, P. (2004). Circulating soluble E-cadherin levels are of prognostic significance in patients with multiple myeloma. Anticancer Res. 24, 2027-2031.

Tattevin, P., Arvieux, C., Dupont, M., Guggenbuhl, P., Lemeur, A., and Michelet, C. (2003). Granulomatous lymphadenitis as a manifestation of Q Fever. Emerg. Infect. Dis. 9, 137-138. doi: 10.3201/eid0901.0 20211

Thuny, F., Textoris, J., Amara, A. B., Filali, A. E., Capo, C., Habib, G., et al. (2012). The gene expression analysis of blood reveals S100A11 and AQP9 as potential biomarkers of infective endocarditis. PLoS ONE 7:e31490. doi: 10.1371/journal.pone.0031490

van Schaik, E. J., Chen, C., Mertens, K., Weber, M. M., and Samuel, J. E. (2013). Molecular pathogenesis of the obligate intracellular bacterium Coxiella burnetii. Nat. Rev. Microbiol. 11, 561-573. doi: 10.1038/ nrmicro3049

Vanlaere, I., and Libert, C. (2009). Matrix metalloproteinases as drug targets in infections caused by gram-negative bacteria and in septic shock. Clin. Microbiol. Rev. 22, 224-239. doi: 10.1128/CMR.00047-08

Voorzanger, N., Touitou, R., Garcia, E., Delecluse, H. J., Rousset, F., Joab, I., et al. (1996). Interleukin (IL)-10 and IL-6 are produced in vivo by nonHodgkin's lymphoma cells and act as cooperative growth factors. Cancer Res. 56, 5499-5505.

Wu, S., Morin, P. J., Maouyo, D., and Sears, C. L. (2003). Bacteroides fragilis enterotoxin induces c-Myc expression and cellular proliferation. Gastroenterology 124, 392-400. doi: 10.1053/gast.2003.50047

Yan, W., Li, S., Wei, M., and Gao, H. (2018). Identification of MMP9 as a novel key gene in mantle cell lymphoma based on bioinformatic analysis and design of cyclic peptides as MMP9 inhibitors based on molecular docking. Oncol. Rep. 40, 2515-2524. doi: 10.3892/or.2018.6682

Zheng, G., Lyons, J. G., Tan, T. K., Wang, Y., Hsu, T. T., Min, D., et al. (2009). Disruption of E-cadherin by matrix metalloproteinase directly mediates epithelial-mesenchymal transition downstream of transforming growth factor- $\beta 1$ in renal tubular epithelial cells. Am. J. Pathol. 175, 580-591. doi: 10.2353/ajpath.2009.080983

Conflict of Interest Statement: The authors declare that the research was conducted in the absence of any commercial or financial relationships that could be construed as a potential conflict of interest.

Copyright (๑ 2019 Mezouar, Omar Osman, Melenotte, Slimani, Chartier, Raoult, Mege and Devaux. This is an open-access article distributed under the terms of the Creative Commons Attribution License (CC BY). The use, distribution or reproduction in other forums is permitted, provided the original author(s) and the copyright owner(s) are credited and that the original publication in this journal is cited, in accordance with accepted academic practice. No use, distribution or reproduction is permitted which does not comply with these terms. 